\title{
SPECT/CT Physical Principles and Attenuation Correction*
}

\author{
James A. Patton ${ }^{1}$ and Timothy G. Turkington ${ }^{2}$ \\ ${ }^{1}$ Vanderbilt University Medical Center, Nashville, Tennessee; and ${ }^{2}$ Duke University Medical Center, Durham, North Carolina
}

\begin{abstract}
Using nuclear medicine techniques, physiologic activity and processes can be identified in a way that is unique from other modalities. Oftentimes it is helpful to know the exact location of the physiologic uptake that is visualized on a scan. Knowing the exact location can sometimes help to distinguish normal from abnormal physiologic uptake. When an abnormality has been identified, knowing the exact location can then be helpful in treatment planning. The ability to provide precise localization of physiologic data from nuclear medicine studies is now possible with hybrid SPECT/CT systems. Additionally, these systems provide an accurate attenuation correction of the nuclear medicine image data. After reading this article, the technologist will be able to list and describe the inherent problems associated with SPECT image acquisition and reconstruction, briefly explain how data acquired from the CT scanner are used to provide attenuation correction data for SPECT and anatomic information for diagnostic purposes, list and briefly describe the different types of clinical SPECT/CT systems, and discuss the importance of accurate CT and SPECT image registration.
\end{abstract}

Key Words: instrumentation; SPECT; SPECT/CT; image fusion; attenuation correction

J Nucl Med Technol 2008; 36:1-10

DOI: 10.2967/jnmt.107.046839

\section{$\mathbf{F}$} or many years, physicians have been faced with the dilemma of identifying a region of increased uptake on a nuclear medicine image and then trying to determine the precise anatomic location of the region. This is of initial importance in differentiating between abnormal uptake and normal physiologic uptake. Once the determination of abnormal uptake has been established, then it is important for physicians to know the exact anatomic location of the region to determine the proper course of therapy. Recent advances in imaging technology have provided physicians with a new and powerful tool to handle this dilemma: integrated SPECT/CT

\footnotetext{
Received Aug. 31, 2007; revision accepted Nov. 7, 2007.

For correspondence or reprints contact: James A. Patton, PhD, Vanderbilt University Medical Center, 21st Avenue South and Garland, Nashville, TN 37232-2675.

E-mail: jim.patton@vanderbilt.edu

*NOTE: FOR CE CREDIT, YOU CAN ACCESS THIS ACTIVITY THROUGH THE SNM WEB SITE (http://www.snm.org/ce_online) THROUGH MARCH 2010. Those without Internet access can obtain a hard copy of the test by calling 703-708-9000, ext. 1247.

COPYRIGHT @ 2008 by the Society of Nuclear Medicine, Inc.
}

systems. This work was pioneered by Lang and Hasagawa et al. (1), who not only combined hardware components into an integrated system but also developed important algorithms for SPECT attenuation correction using CT images. This technology makes it possible to acquire physiologic and anatomic images in a registered format and fuse them so that precise anatomic localizations of radiopharmaceutical distributions can readily be visualized. An additional benefit of this technologic advance is that the anatomic images can be used to perform high-quality attenuation corrections of the radiopharmaceutical distributions. To properly acquire these registered images and identify potential pitfalls in the process, it is necessary to have a good working knowledge of the physical principles and instrumentation involved in the data acquisition and image reconstruction processes.

\section{SPECT}

Data required for constructing images using the techniques of SPECT are acquired by collecting planar images (typically in $64 \times 64$ or $128 \times 128$ data-point arrays) at multiple angles around the patient using single-, dual-, or triple-head scintillation cameras typically equipped with parallel-hole collimators, which provide projection views of the radioactivity from those angles. Typically, 120 images are acquired at $3^{\circ}$ increments for $360^{\circ} \mathrm{SPECT}$ (60 images at $3^{\circ}$ increments for $180^{\circ}$ SPECT in cardiac imaging), as shown in Figure $1 \mathrm{~A}$ for a simulated acquisition from a uniform elliptic phantom. A single line of data in a planar projection image is a count profile of data acquired from a slice of activity, and corresponding lines (count profiles) are filtered and backprojected into an image space (Fig. 1B) to construct an image of a transverse slice of the activity distribution as an array of counts at that slice location, as shown in Figure 1C. Images acquired with SPECT are often used in oncologic applications and provide physiologic information based on localization of radiopharmaceuticals in regions of interest. However, these images suffer from poor spatial resolution (typically $>$ $1 \mathrm{~cm}$ ) and often lack anatomic landmarks for precise determinations of location of areas of abnormal uptake. In addition, normal physiologic distributions must often be differentiated from regions of abnormal uptake, and this is difficult without anatomic landmarks for accurate correlations. Another problem inherent in SPECT is illustrated in Figure 1D. This is a representation of the image that would 


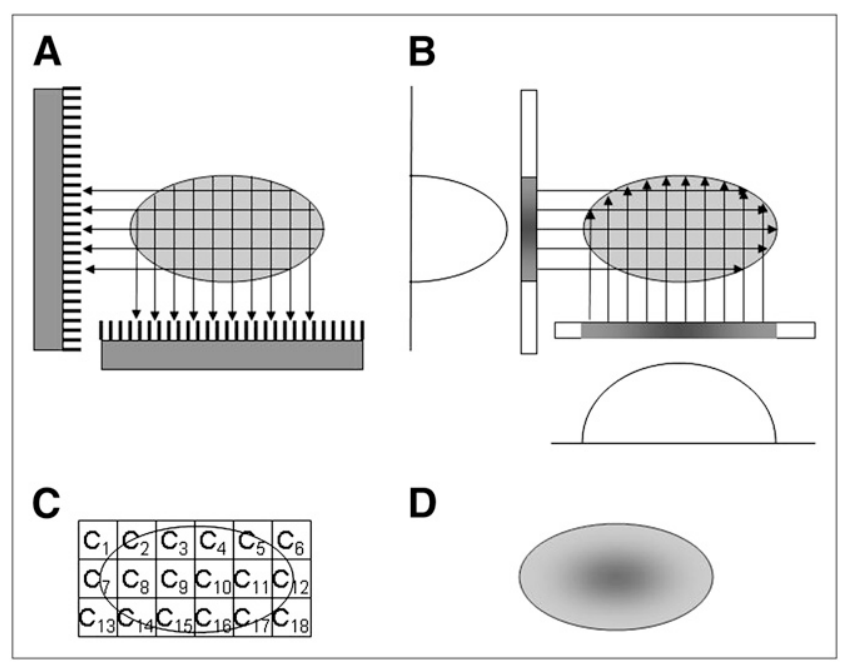

FIGURE 1. (A) Conventional SPECT techniques use scintillation camera to acquire projection images around patient in orthogonal geometry. (B and $\mathrm{C}$ ) Count profiles are filtered and backprojected into image space $(B)$ to produce reconstructed arrays of counts representing slices of activity from region of interest (C). (D) These images are not accurate representations of actual distribution because of attenuation effects.

result from a reconstruction of the data acquisition process illustrated in Figure 1B. Even though the source distribution is uniform, the reconstructed image shows an apparent decrease in activity that reaches a minimum at the center of the image. This effect is due to attenuation of photons within the source (i.e., the human body) before exiting the source and being detected by the camera system $(2,3)$.

The primary mechanism for attenuation in tissue throughout the diagnostic energy range in conventional nuclear medicine is Compton scattering, which results in changes in photon directions of travel with loss of energy. The change of direction of a photon may result in a missed count, if the photon had an initial trajectory that would have passed through the collimator but the change in direction prevented it from being detected. In other cases, the scattered photon is detected (in the wrong location), leading to a false background. Figure 2A illustrates the magnitude of attenuation effects in nuclear medicine with singlephoton emitters (4). This figure shows the percentage transmission of photons as measured with a single planar acquisition from a point source as a function of depth in a $20-\mathrm{cm}$ cylindric water phantom for radionuclides commonly used in nuclear medicine applications. The effects of attenuation are more intense at lower energies but are still significant at the highest energy value $\left(364 \mathrm{keV}\right.$ for $\left.{ }^{131} \mathrm{I}\right)$. In addition, the magnitude of the attenuation effect depends on the tissue type, as shown in Figure 2B (4). This figure shows the percentage transmission of $140-\mathrm{keV}$ photons versus depth in lung, soft tissue, and bone. The effect of this attenuation problem in SPECT applications is shown in Figure 3. The data in this figure were calculated from the data in Figure 2B by summing 2 planar projection images

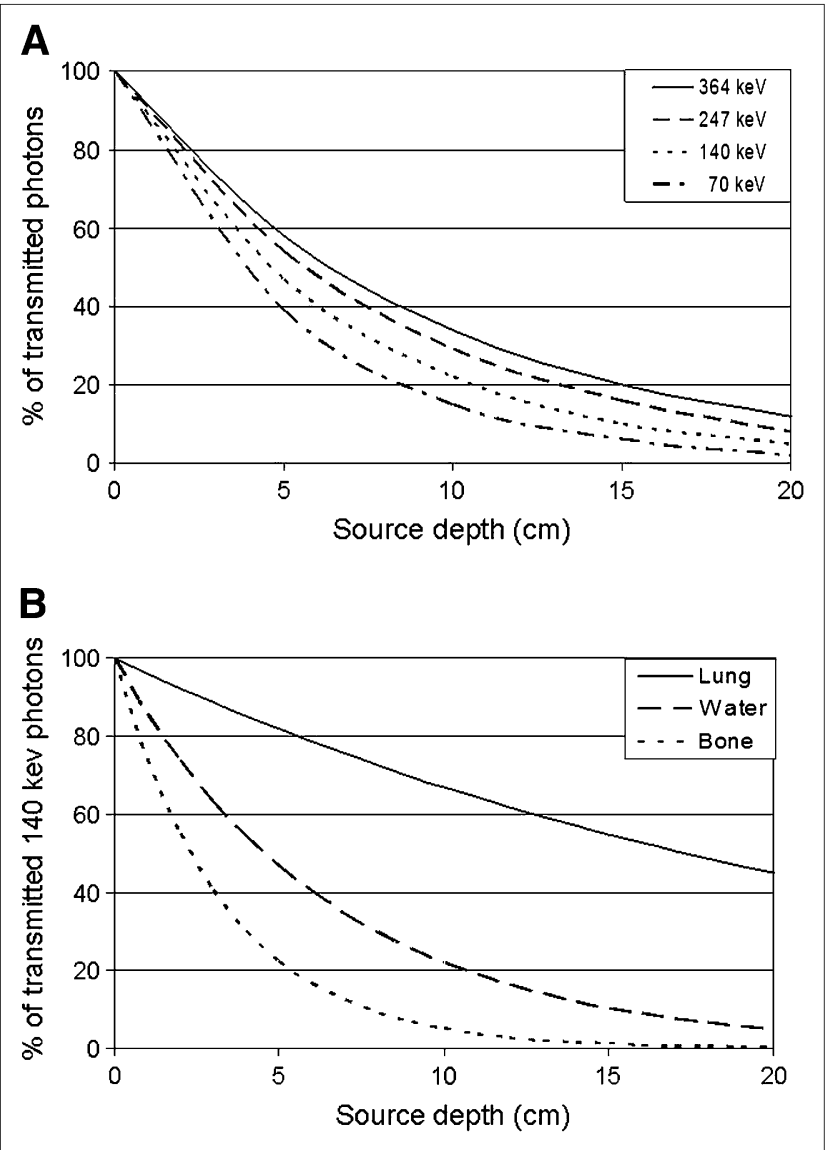

FIGURE 2. (A) Plot of percentage of transmitted photons as function of depth in $20-\mathrm{cm}$ cylindric phantom measured in single planar projection image for radionuclides commonly used in nuclear medicine. (B) Plot of percentage of transmitted photons from ${ }^{99 \mathrm{~m} T c}$ source as function of depth in lung, water, and bone.

separated by $180^{\circ}$. Thus, to accurately represent the activity distribution measured with SPECT, it is necessary to accurately correct for the effects of attenuation.

\section{CT}

The deficiencies in SPECT identified above can be addressed by incorporating the techniques of $\mathrm{CT}$ in the image acquisition and reconstruction process. CT provides highquality and high-spatial-resolution $(\sim 1 \mathrm{~mm})$ images of cross-sectional anatomy. CT provides a significant portion of the anatomic images acquired in oncologic applications, not only for diagnosis and staging of disease but also for simulations used to plan for radiation therapy. CT images have a high sensitivity for lesion detection but often have limited specificity. In addition, it is often difficult to differentiate between tumor recurrence and posttreatment changes in these anatomic images. CT images are acquired as transmission maps with a high photon flux and are actually highquality representations of tissue attenuation and thus can provide the basis for attenuation correction. Therefore, combining SPECT and CT modalities into an integrated 


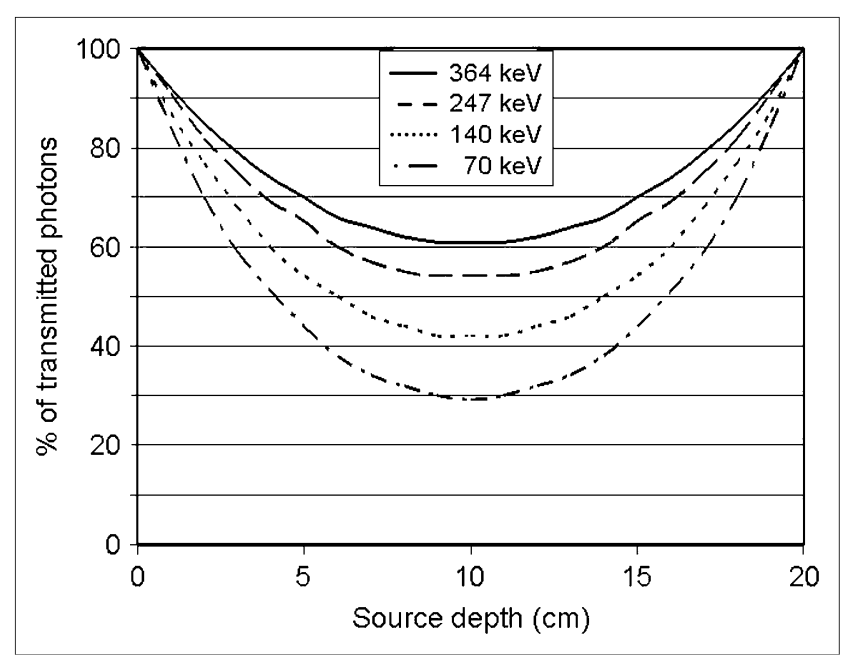

FIGURE 3. Plot of transmitted photons as function of depth in $20-\mathrm{cm}$ cylindric phantom calculated from data in Figure $2 \mathrm{~A}$ by summing 2 planar projection images separated by $180^{\circ}$.

system is a significant advance because the 2 modalities are complementary in that the weaknesses of one are often the strengths of the other in specific imaging situations.

$\mathrm{CT}$ images are acquired by using a high-output $\mathrm{x}$-ray tube and an arc of detectors in a fixed geometry to acquire crosssectional transmission images of the patient as the $\mathrm{x}$-ray tube and detector configuration rapidly rotates around the patient as shown in Figures 4A and 4B. Current technology permits the simultaneous acquisition of as many as 64 thin slices $(0.625 \mathrm{~mm}$ ) in as little as $0.35 \mathrm{~s}$ (If the rotation is $0.35 \mathrm{~s}$, only a little more than half the rotation is actually required to produce images). The geometry of these third-generation CT scanners results in the acquisition of transmission data in a fanbeam geometry. However, it can be shown that each ray in a fanbeam geometry can be represented by an equivalent ray in a parallel-beam geometry. Therefore, a common approach is to convert the fanbeam data to parallel-beam geometry as illustrated diagrammatically in Figures $4 \mathrm{C}$ and $4 \mathrm{D}$, to simplify the reconstruction process. As many as 600 projection arrays are acquired in this manner to produce a high-quality transmission measurement of each slice of tissue.

Each measured ray $(I)$ is the initial ray intensity $\left(I_{0}\right)$ altered by an attenuation factor:

$$
I=I_{0} e^{\sum_{i}-\mu_{i} x_{i}},
$$

where the index $i$ represents all the different tissue type regions along the trajectory, $\mu_{i}$ are the effective attenuation coefficients for the different tissue region, and $x_{i}$ are the corresponding thicknesses of the tissue regions, so that the sum represents the total attenuation through all regions. With filtered backprojection (or another tomographic reconstruction technique), these attenuation measurements obtained along all rays at all angles are used to produce a

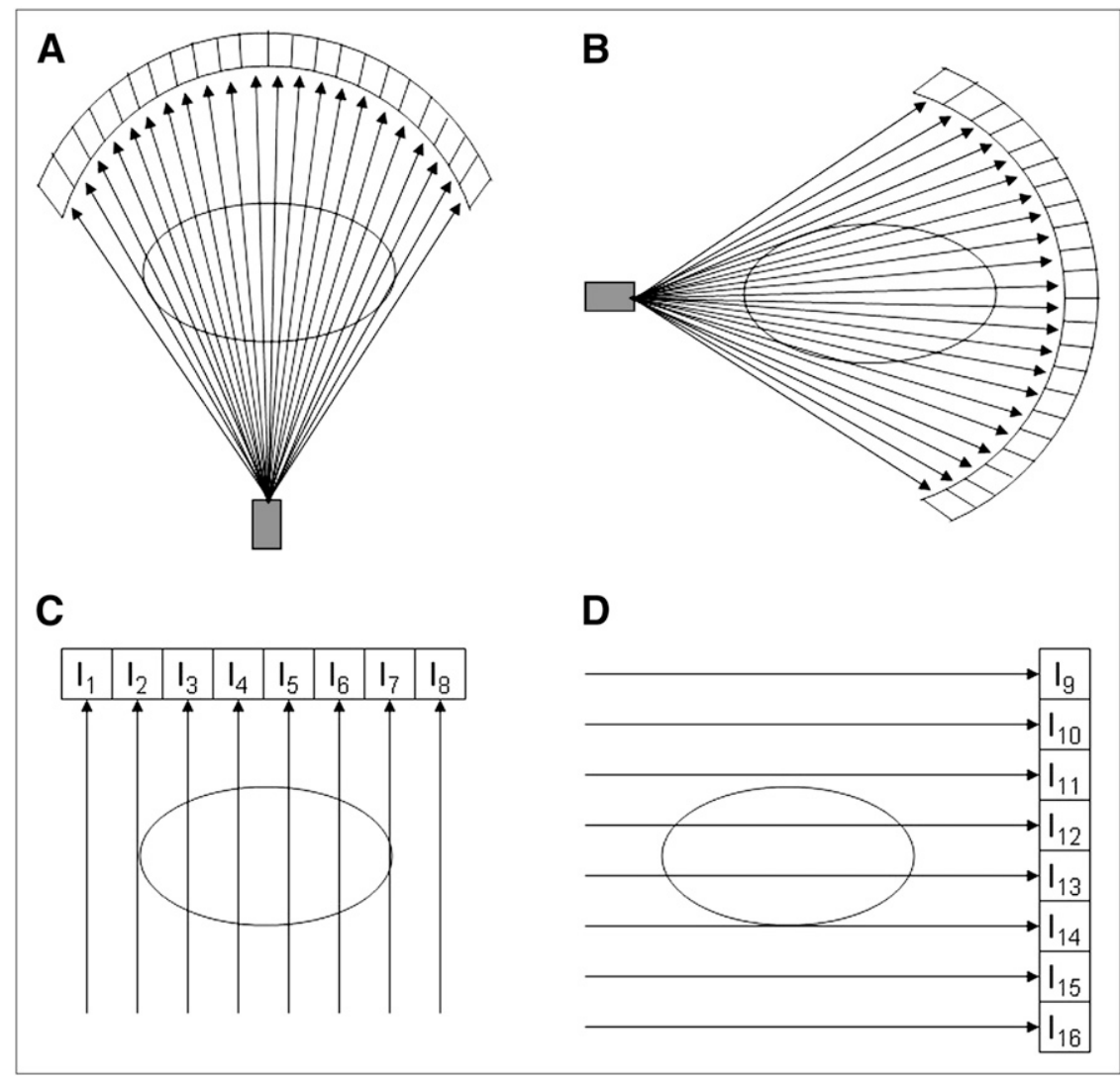

FIGURE 4. (A and B) CT data are acquired in fanbeam geometry where individual rays represent transmitted photon intensities from multiple projections around patient. ( $C$ and $D$ ) These data can be reformatted into orthogonal geometry similar to that used for SPECT. 
cross-sectional array of tissue attenuation coefficients as shown in Figure 5A. The resulting array is a high-quality image of body attenuation and therefore is representative of body anatomy. To standardize the data and provide a sufficient gray scale for display, the data are typically converted to CT numbers (Hounsfield units), as shown in Figure 5B, by normalizing to the attenuation coefficient of water using the following equation:

$$
\text { CT number }=\left[\left(\mu_{\text {tissue }}-\mu_{\text {water }}\right) / \mu_{\text {water }}\right] \times 1,000 \text {. }
$$

Based on this convention, the CT numbers of air and water are $-1,000$ and 0 , respectively. These images are typically displayed as $256 \times 256$ or $512 \times 512$ arrays, with pixels representing $0.5-2.0 \mathrm{~mm}$ of tissue, because of the high spatial resolution inherent in the measurements $(5,6)$.

\section{SPECT/CT}

The integration of SPECT and CT systems into a single imaging unit sharing a common imaging table provides a significant advance in technology because this combination permits the acquisition of SPECT and CT data sequentially in a single patient study with the patient in an ideally fixed position. Thus, the 2 datasets can be acquired in a registered format by appropriate calibrations, permitting the acquisition of corresponding slices from the 2 modalities. The CT data can then be used to correct for tissue attenuation in the SPECT scans on a slice-by-slice basis. Because the CT data are acquired in a higher-resolution matrix than the SPECT data, it is necessary to decrease the resolution of the CT data to match that of SPECT. In other words, the CT data are blurred to match the SPECT data. From the attenuation coefficient data acquired with $\mathrm{CT}$, correction factors can then be determined as shown in Figure 6B, which can then be used to correct the SPECT data (Fig. 6A) for attenuation, yielding the attenuation-corrected SPECT data as shown in Figure 6C $(4,7)$.

One additional topic must be addressed to ensure the accuracy of the attenuation correction. The output of the $\mathrm{X}$-ray tube used in $\mathrm{CT}$ provides a spectrum of photon energies from $0 \mathrm{keV}$ up to the maximum photon energy $(\mathrm{kVp}=$ peak

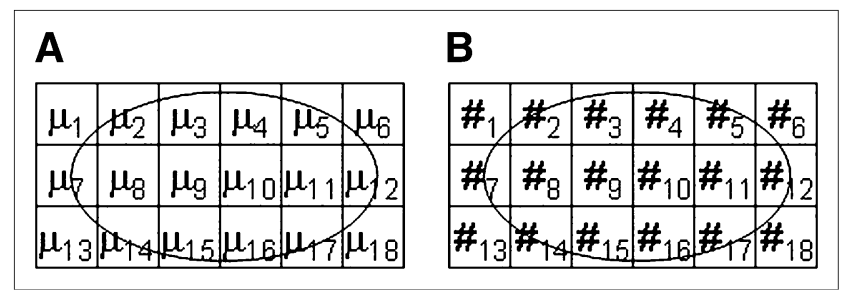

FIGURE 5. Transmitted intensities can be used to solve for attenuation coefficients $(\mu)$ by using unattenuated intensity $\left(\mathrm{I}_{\mathrm{o}}\right)$ by attenuation equation $\left(I=I_{0} e^{-\mu x}\right)$. (A and $B$ ) Using filtered backprojection, array of attenuation coefficients for each anatomic slice can be determined $(A)$ and converted to array of CT numbers for display purposes (B). energy in keV) setting used for the acquisition, as shown in Figure 7. Because low-energy photons are preferentially absorbed in tissue, the beam spectrum shifts toward the higher energy end as it passes through more tissue, thereby changing its effective $\mu$ and producing a variety of artifacts (beam-hardening effects) in images, and filtering of the beam to remove low-energy photons is required. The spectrum after filtering, shown in Figure 7, has been "hardened" to reduce these effects. The resulting spectrum has an effective energy (mean) of approximately $70 \mathrm{keV}$ in the example in Figure 7. Because attenuation effects vary with energy, as shown in Figure 2, it is necessary to convert the attenuation data acquired with CT to match the energy of the radionuclide used in the SPECT acquisitions. For the example in Figure 7, it is necessary to convert the attenuation data measured at an effective energy of $70 \mathrm{keV}$ to $140 \mathrm{keV}$ for ${ }^{99 \mathrm{~m}}$ Tc. This is typically accomplished by using a bilinear model (8-10) relating attenuation coefficients at the desired energy to CT numbers measured at the effective energy of the CT beam of x-rays, as shown in Figure 8. For CT numbers less than 0 , the measured tissue is assumed to be a combination of air and water, and the attenuation coefficient at the desired energy $(140 \mathrm{keV})$ can be calculated from the CT number by the following equation:

$$
\mu_{\text {tissue, } 140 \mathrm{keV}}=\frac{\mathrm{CT} \# *\left(\mu_{\mathrm{water}, 140 \mathrm{keV}}-\mu_{\mathrm{air}, 140 \mathrm{keV}}\right)}{1,000} .
$$

This equation describes the first component of the bilinear curve in Figure 8. For CT numbers greater than 0, the conversion is more complicated because the measured tissue is a combination of water and bone. In this case, the attenuation coefficient at the desired energy $(140 \mathrm{keV})$ can be calculated from the CT number by the following equation:

$$
\begin{aligned}
& \mu_{\text {tissue, } 140 \mathrm{keV}}=\mu_{\text {water, } 140 \mathrm{keV}} \\
& +\frac{\mathrm{CT} \# * \mu_{\text {water,keVoff }} *\left(\mu_{\mathrm{bone}, 140 \mathrm{keV}}-\mu_{\text {water, } 140 \mathrm{keV}}\right)}{1,000 *\left(\mu_{\mathrm{bone}, \mathrm{keV} \text { off }}-\mu_{\text {water,keVoff }}\right.} .
\end{aligned}
$$

This equation describes the second component of the bilinear curve in Figure 8. In practice, the various attenuation coefficients for specific photon energy used in the SPECT acquisition and the effective photon energy used in the CT acquisition can be found in a stored look-up table in the reconstruction algorithm. The conversion can then be performed using 2 simple linear relationships relating the attenuation coefficient at the desired energy and the measured CT numbers for specific measured tissues.

There are numerous advantages in the use of CT data for attenuation correction of emission data. First, the CT scan provides a high photon flux that significantly reduces the statistical noise associated with the correction in comparison to other techniques (i.e., radionuclides used as transmission sources). Also, because of the fast acquisition speed of CT scanners, the total imaging time is significantly 


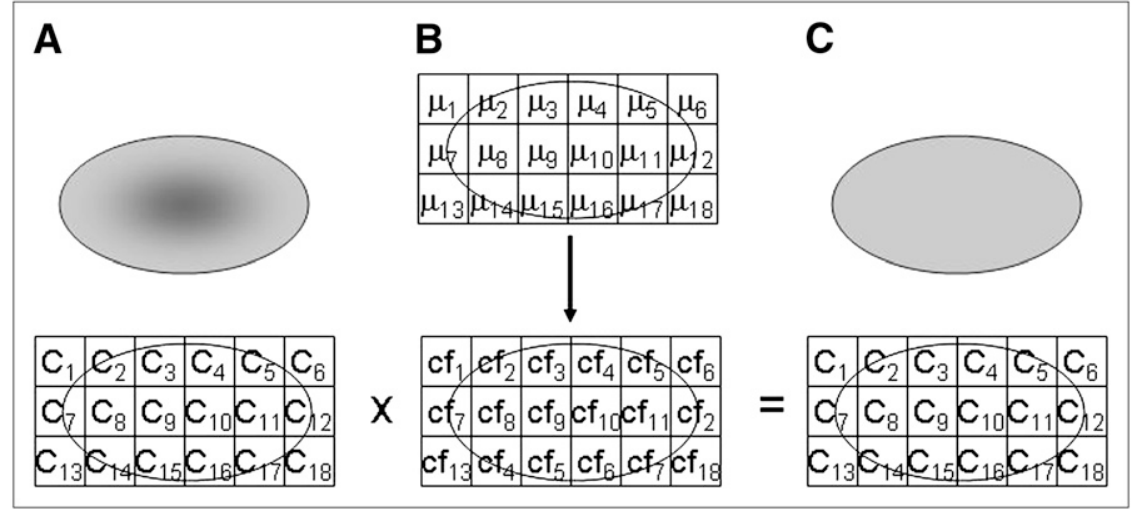

FIGURE 6. Array of attenuation correction factors (B) can be determined from attenuation coefficient measurements determined from CT scan and used to correct emission counts from uncorrected SPECT scan $(A)$ to provide final attenuation-corrected SPECT scan (C). reduced by using this technology. Another advantage related to the high photon flux of CT scanners is that attenuation measurements can be made in the presence of radionuclide distributions with negligible contributions from photons emitted by the radionuclides (i.e., postinjection CT measurements can be performed). The use of CT also eliminates the need for additional hardware and transmission sources that often must be replaced on a routine basis. And of course the anatomic images acquired with CT can be fused with the emission images to provide functional anatomic maps for accurate localization of radiopharmaceutical uptake.

An example of the image fusion process is shown in Figure 9. These are images from a patient with hyperparathyroidism caused by the presence of a parathyroid adenoma. The adenoma was located in the mediastinum, as demonstrated by the planar images shown in Figure 9A. Separate SPECT and CT scans were acquired sequentially in registered format from the region of interest using an integrated SPECT/CT system. The low-resolution SPECT images (Fig. 9C) were interpolated into a data matrix size comparable to the corresponding CT images (Fig. 9B). The SPECT images were then displayed with a semitransparent color scale and super-

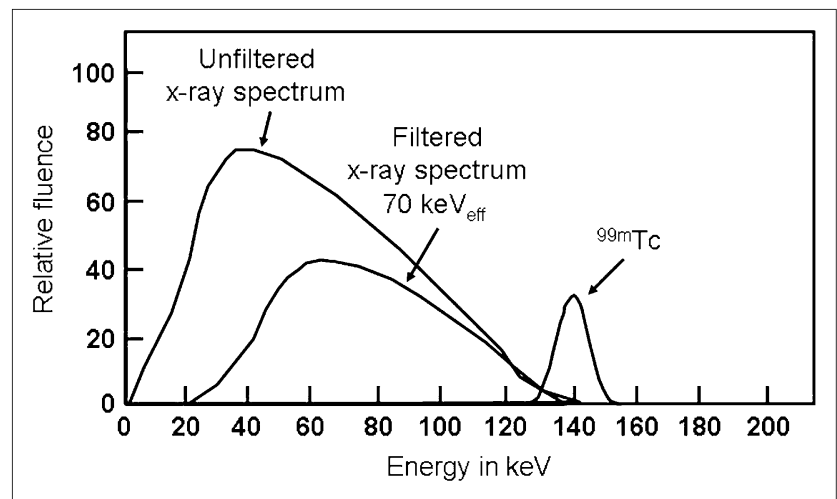

FIGURE 7. Typical energy spectrum of x-rays from x-ray tube. Filtered curve shows effects of filtration (beam hardening), which is used for CT. These data can be applied for attenuation correction of single-photon emitters such as $99 \mathrm{mTc}$ using bilinear model shown in Figure 8. imposed with the gray-scale CT images so that the 2 distributions can be displayed as combined images as shown in Figure 9D. Only transverse and coronal data are shown here, but complete sets of transverse, sagittal, and coronal views were available for image interpretation. These datasets are typically displayed with cursor control so that they can easily be reviewed by the interpreting physician. Current clinical experiences have shown that the use of SPECT/CT provides the interpreting physician with a higher level of confidence in providing diagnostic information, and the presence of anatomic data from CT in fused images typically adds diagnostic information in $30 \%-35 \%$ of the cases, with even higher contributions in specific disease processes (11).

\section{ACCURACY OF CT ATTENUATION CORRECTION}

The accuracy of attenuation correction of SPECT scans with CT transmission measurements was measured using a dual-head scintillation camera (Millennium VG) equipped with a low-output, single-slice CT scanner (Hawkeye) manufactured by General Electric Healthcare. Six radionuclidecollimator combinations were evaluated as shown in Table 1. A water-filled thorax phantom was used for the study with 2 identically prepared vials containing a solution of each of the radionuclides, as shown in Figures 10A and 10B. One vial

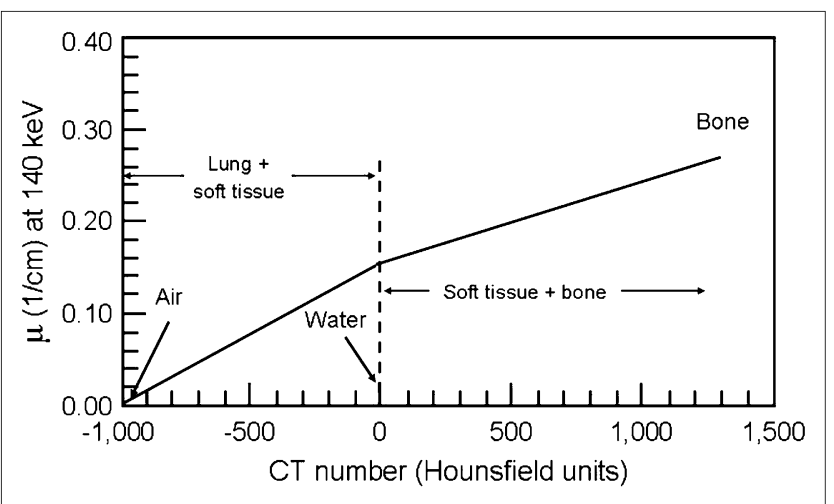

FIGURE 8. Bilinear model commonly used for converting measured CT numbers to attenuation coefficients for specific radionuclide such as ${ }^{99 \mathrm{~m}} \mathrm{Tc}$. 

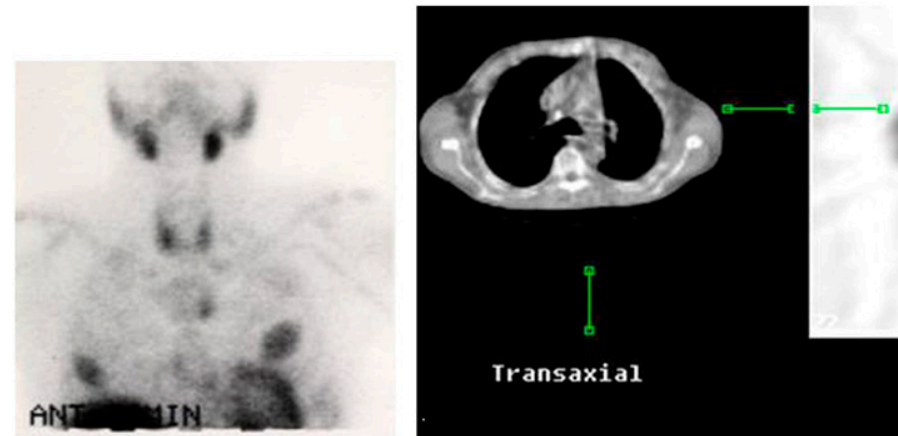

Transaxial
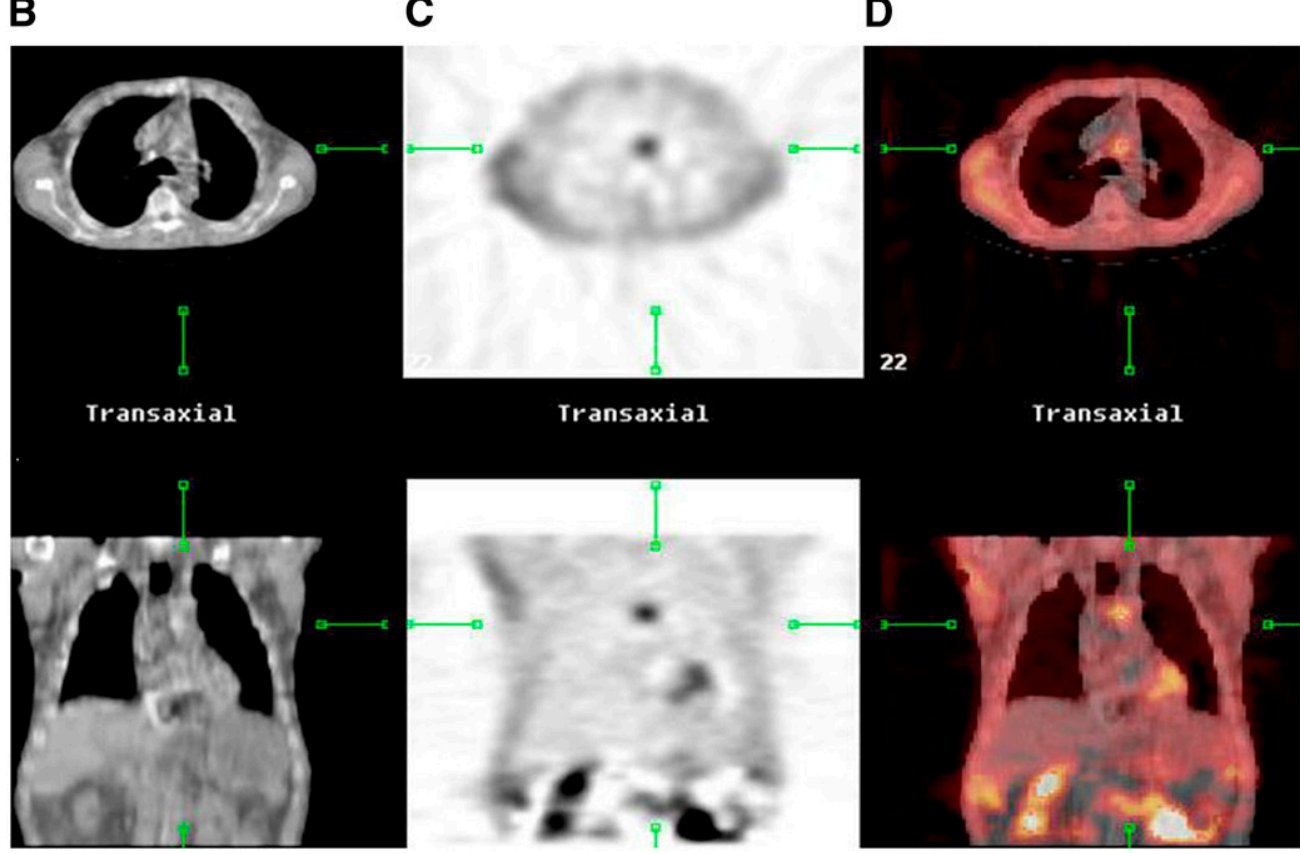

Transaxial

22

FIGURE 9. (A) Anterior and lateral planar ${ }^{99 m}$ Tc-sestamibi images of patient with parathyroid adenoma. SPECT/CT study with GE Infinia-Hawkeye demonstrated anatomy of region of interest with CT (B) in transverse and coronal images. (C) Corresponding transverse and coronal images of emission distribution with SPECT clearly identified presence of adenoma. (D) Fused images of 2 datasets provided precise anatomic location of lesion in left mediastinum. Availability of multiple fused images from transverse, sagittal, and coronal planes provided surgeon with accurate anatomic roadmap for use in operating room.

was placed inside the water-filled phantom for attenuation correction measurements, and the second vial was placed outside the phantom in line with the first vial, as shown in Figure 10C, and was used as the standard (i.e., no external attenuation). Sequential SPECT and CT scans were obtained with each of the radionuclide-collimator combinations, and the SPECT scans were corrected for attenuation using the CT data. Images were reconstructed using an iterative reconstruction algorithm (ordered-subsets expectation maximization with 2 iterations). Figure 10D shows coronal slices through the vials and corresponding count profiles without and with attenuation correction for the study conducted with ${ }^{99 \mathrm{~m}} \mathrm{Tc}$. Regions of interest were defined to surround each vial, and regions of interest from all slices in which the vials were visualized were summed to obtain total measured counts for the attenuation-corrected scans and the noncorrected scans. These summed counts were normalized to the counts mea-

TABLE 1

Collimator and Radionuclide Combinations for CT Attenuation Correction Evaluation

\begin{tabular}{lcl}
\hline Radionuclide & Energy & \multicolumn{1}{c}{ Collimator } \\
\hline${ }^{201} \mathrm{Tl}$ & $70 \mathrm{keV}$ & Low-energy, high-resolution \\
${ }^{99 \mathrm{~m} T \mathrm{Tc}}$ & $140 \mathrm{keV}$ & Low-energy, high-resolution \\
${ }^{111} \mathrm{In}$ & 172 and $247 \mathrm{keV}$ & Medium energy \\
${ }^{131} \mathrm{I}$ & $364 \mathrm{keV}$ & Medium energy \\
${ }^{131} \mathrm{I}$ & $364 \mathrm{keV}$ & High energy \\
${ }^{18} \mathrm{~F}$ & $511 \mathrm{keV}$ & High energy \\
\hline
\end{tabular}

sured from the external vial $(100 \%)$. The results of these measurements are shown in Figure 11. Without attenuation correction, the attenuation of the internal vials ranged from $60 \%$ for ${ }^{18} \mathrm{~F}$ to $72 \%$ for ${ }^{201} \mathrm{Tl}$. The data with attenuation correction show that the internal ${ }^{201} \mathrm{Tl}$ vial was overcorrected by $6 \%$ and the remaining vials were undercorrected by values ranging from $5 \%$ for the ${ }^{131}$ I vial imaged with the mediumenergy collimator to $12 \%$ for the ${ }^{131} \mathrm{I}$ vial and the ${ }^{18} \mathrm{~F}$ vial imaged with the high-energy collimator. It should be noted that a significant overcorrection of a ${ }^{201} \mathrm{Tl}$ image could result in the masking of a true defect.

A second set of measurements was obtained as shown in Figure 12 to evaluate the effect of source location on the accuracy of the attenuation correction. Two identically prepared 2-cm-diameter spheric sources were filled with a solution of ${ }^{99 \mathrm{~m}} \mathrm{Tc}$. One source was placed inside the phantom and the second source was placed outside the phantom in line with the first source. Two phantoms were used and 3 source locations with each phantom. The first phantom was a uniform 20-cm-diameter water-filled phantom, and the 3 source locations are shown in Figures 12A-12C. The second source was a thorax phantom with simulated organs, and the sources were placed adjacent to a lung, in the center of the mediastinum, and adjacent to the spine, as shown in Figures 12D-12F. Sequential SPECT scans with a low-energy, highresolution collimator and CT scans were performed for each phantom-source geometry, and the SPECT scans were corrected for attenuation using the CT data. The SPECT scans without attenuation correction were reconstructed 


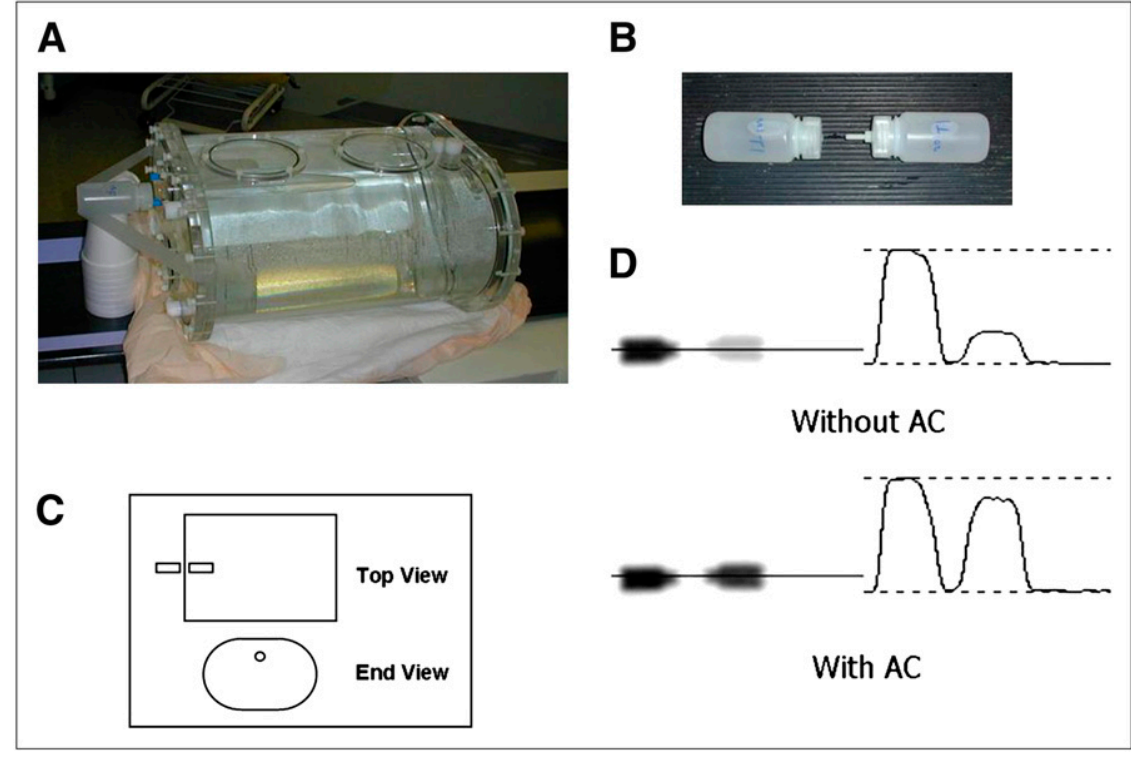

FIGURE 10. (A-C) Phantom configuration used for accuracy verification of CT attenuation correction techniques. (D) Count profiles of uncorrected and attenuation-corrected data with ${ }^{99 \mathrm{~m} T \mathrm{~T} \text {. }}$ using filtered backprojection, and the SPECT scans with attenuation correction were reconstructed using an orderedsubsets expectation maximization algorithm with 2 iterations. Regions of interest were identified and total measured counts determined as in the first experiment, and the data were normalized to the external source measurements $(100 \%)$. The results are shown in Figure 13 for the 6 geometries. For the cylindric phantom, the attenuation of the internal sources ranged from $53 \%$ to $73 \%$. The attenuationcorrected values ranged from $-3 \%$ to $2 \%$. For the thorax phantom, the attenuation of the sources ranged from $61 \%$ to $83 \%$. The data with attenuation correction show that the source adjacent to the spine was overcorrected by $6 \%$ and the remaining 2 sources were undercorrected by $13 \%-15 \%$. The data were also processed using ordered-subsets expectation maximization reconstruction with 2 iterations for the uncorrected images and ordered-subsets expectation maxi-

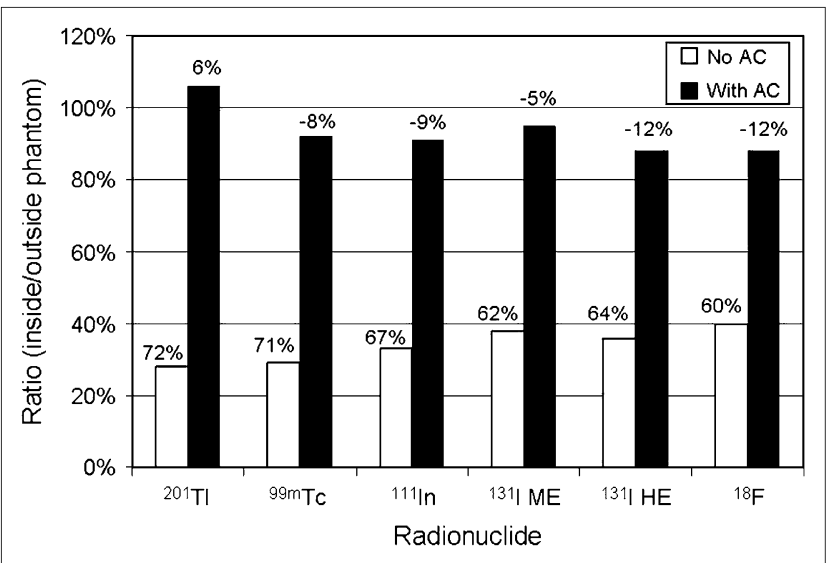

FIGURE 11. Plot of ratio of uncorrected and attenuationcorrected activity measurements for radionuclides used in attenuation correction validation experiment. mization with 4 iterations for the corrected images, and these changes did not alter the resulting measurements.

In addition to attenuation effects, SPECT quantitative accuracy can be degraded by other effects, including spatial resolution effects and scattered events. These studies were designed to isolate the attenuation effects as much as possible. Scatter effects were minimized by not having extended radioactivity distributions, so that there was little radiation to scatter into the regions being measured. Spatial resolution effects were controlled by using identically sized bottles or spheres and using an objective region-of-interest methodology.

From these 2 experiments it can concluded that the application of attenuation data acquired by CT can be used to adequately correct for attenuation effects in routine clinical SPECT where the common practice is to compare regions in the same body section. However, if it is desired to make absolute quantitation measurements, additional corrections should be performed. These may include scatter corrections, partial-volume corrections, and corrections for collimator response. In addition, accurate calibrations of measured counts versus activity for the system, a process requiring blood sampling and not typically done in routine $\gamma$-camera use, would need to be implemented (12).

\section{CLINICAL SPECT/CT SYSTEMS}

Clinical SPECT/CT systems currently available from manufacturers typically have dual-head scintillation cameras positioned in front of the CT scanner and sharing a common imaging table. There are 2 approaches to clinical SPECT/CT applications. The first approach is the use of a low-output, slow-acquisition CT scanner, the Hawkeye with dual-head Infinia manufactured by General Electric Healthcare Systems. The CT scanner consists of a low-output x-ray tube $(2.5 \mathrm{~mA})$ and 4 linear arrays of detectors and can acquire four 

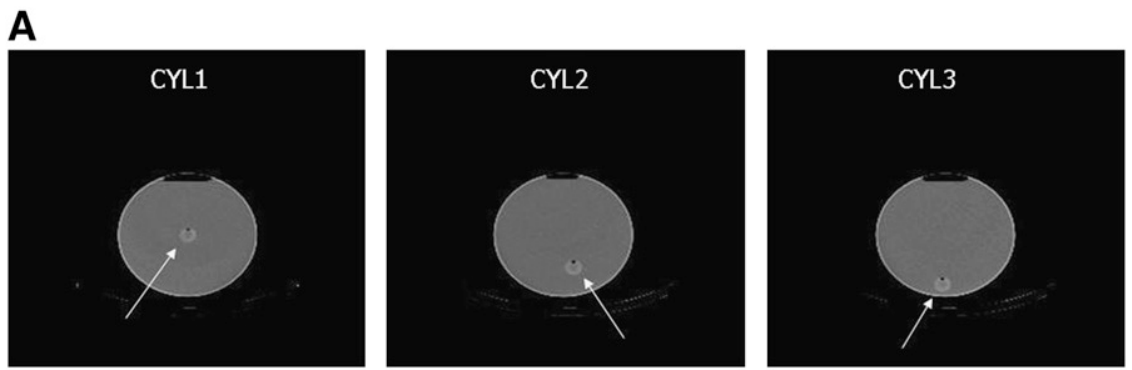

B
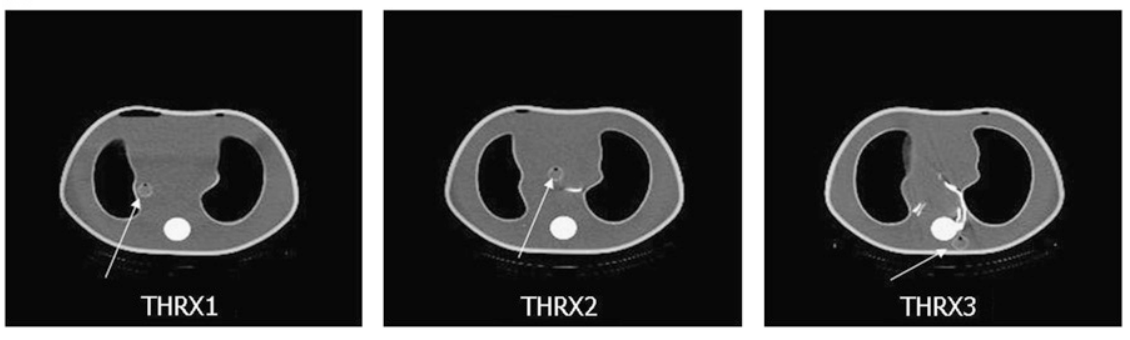

FIGURE 12. Phantom configurations using $99 \mathrm{mT}$ Tc spheric sources for evaluation of accuracy of attenuation correction as function of lesion location. Arrows show lesion location in $20-\mathrm{cm}$ cylindric phantom (A) and thorax phantom (B).

5-mm anatomic slices in $13.6 \mathrm{~s}$ with a high-contrast spatial resolution of more than $3 \mathrm{lp} / \mathrm{cm}$. The images acquired with this system are not of sufficient quality to be used for billable procedures but are sufficient to be used for attenuation correction and anatomic correlation with emission scans. The slow scan speed is actually an advantage in regions where there is physiologic motion because the CT image blurring from the motion is comparable to that of the emission scans, resulting in a good match in fused images. The radiation dose from this system is typically less than $5 \mathrm{mGy}(500 \mathrm{mrad})$, compared with values of $10-100 \mu \mathrm{Gy}(1-$ $10 \mathrm{mrad}$ ) for applications using radioisotope transmission sources.

The second approach is to integrate commercially available CT scanners with dual-head scintillation cameras. The Symbia with dual-head E-Cam manufactured by Siemens Medical Systems is available in 1-, 2-, and 6-slice versions with variable tube currents (20-345 mA), slice thicknesses of $0.6-10 \mathrm{~mm}$, and rotational speeds of $0.6-1.5 \mathrm{~s}$. The Precedence with dual-head Skylight manufactured by Philips is

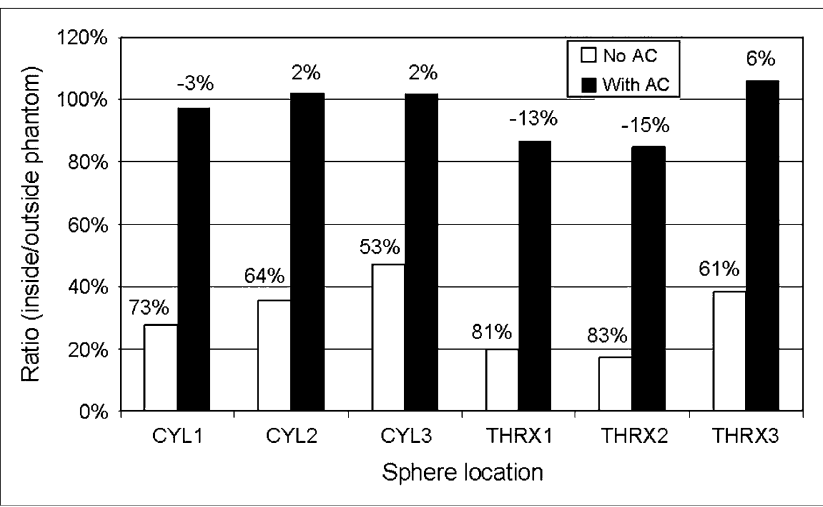

FIGURE 13. Plot of ratio of uncorrected and attenuationcorrected activity measurements for phantom configurations shown in Figure 12 using ${ }^{99 m} \mathrm{Tc}$. available in 16- and 40-slice versions with variable tube currents (20-500 mA), slice thicknesses of 0.6-12 mm, and rotational speeds as fast as $0.5 \mathrm{~s}$. These systems exhibit highcontrast spatial resolutions of $13-15 \mathrm{lp} / \mathrm{cm}$ with approximately 4-5 times the patient radiation dose of that from the Hawkeye system. Because the CT scanners in the systems are commercially available diagnostic systems, the images produced are of sufficient quality to be used for billable procedures, in addition to the obvious attenuation correction and anatomic correlation applications. Radiation doses from these systems are on the order of 20 mGy ( 2 rads) when techniques are used for diagnostic-quality image production. It should be noted that these systems can also be operated in a lower-radiation-dose mode by reducing the $\mathrm{x}$-ray tube current. Although the images provided by this mode of operation typically are not of sufficient quality to be used for billable procedures, they are acceptable for attenuation correction and anatomic correlation applications.

Because the radiation dose from each of these approaches is not trivial, the radiation dose from the CT scan, whether for diagnostic purposes or only for attenuation correction applications, must be considered in individual imaging applications.

A new research-prototype SPECT/CT system has been designed specifically for cardiac imaging applications. This system was manufactured by General Electric Healthcare Systems and integrates the Ventri dual-head, fixed $90^{\circ}$ geometry cardiac camera with the 64 slice VCT CT scanner and is being evaluated at Vanderbilt University Medical Center. This system provides the capability of cardiac perfusion scanning, calcium scoring, and CT angiography in a single combined-imaging procedure. An example of this combined procedure is shown in Figure 14. These images were obtained from a patient who had a history of coronary artery disease with a subtotal occlusion of the left anterior descending coronary artery (LAD) and had a left internal 
A

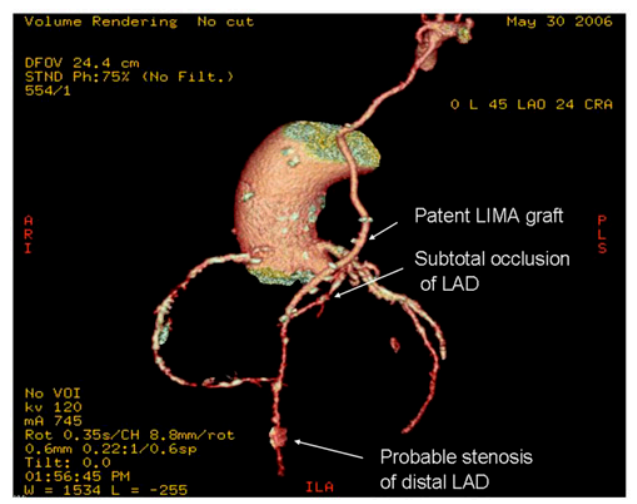

B

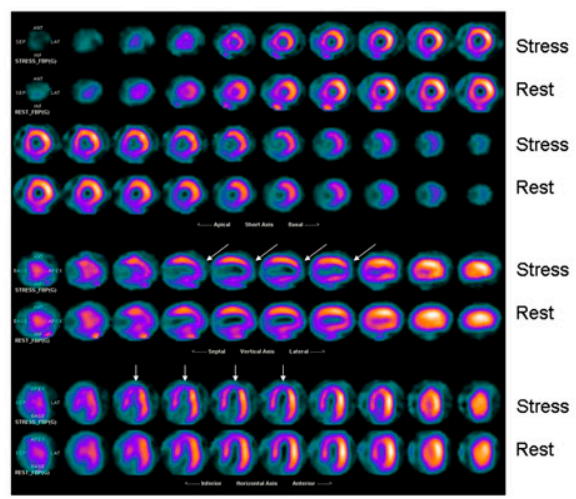

C

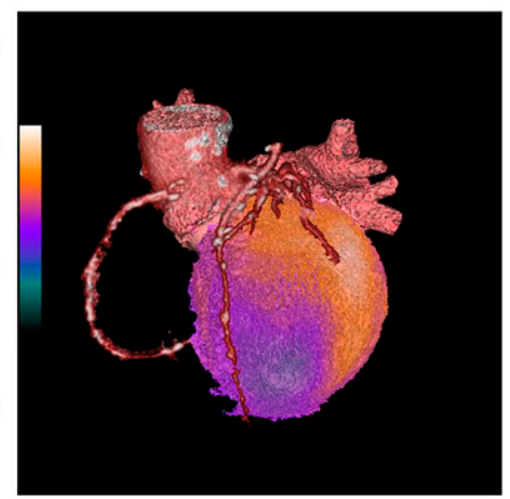

FIGURE 14. (A) CT angiogram of patient demonstrating multiple calcium deposits, patent left internal mammary artery graft, previously diagnosed subtotal occlusion of LAD, and newly identified probable stenosis of distal LAD. (B) Attenuation-corrected rest and stress perfusion study showing new region of ischemia in apex (arrows). Rows $1+3$ and $2+4$ are short-axis stress and rest images, respectively. Rows 5 and 6 are vertical long-axis stress and rest images, respectively. Rows 7 and 8 are horizontal long-axis stress and rest images, respectively. (C) Three-dimensional fusion of CT angiogram and stress perfusion studies demonstrating correlation between probable stenosis of distal LAD and region of ischemia in apex.

mammary artery graft procedure in the past. He presented with renewed exertional chest pain. The CT angiogram (Fig. 14A) showed the left internal mammary artery graft to be patent, identified the subtotal occlusion of the LAD, and in addition identified a probable stenosis of the distal LAD. Numerous calcium deposits were also clearly seen in this image. The attenuation-corrected stress perfusion study performed with ${ }^{99 \mathrm{~m}} \mathrm{Tc}$-sestamibi demonstrated a new region of ischemia in the apex in addition to regions previously identified (arrows), as shown in Figure 14B. Because the data from these separate imaging procedures were acquired in registered format, surface rendering techniques were used to produce 3-dimensional images, which were then fused to produce the image shown in Figure 14C. This image demonstrates the correlation between the stenosis in the distal LAD and the ischemic region in the apex.

\section{QUALITY ASSURANCE}

In addition to performing the quality assurance procedures that are routine with CT scanners and scintillation cameras with SPECT capability, it is important to routinely verify the accuracy of the registration techniques that are used with these combined systems. Errors in registration will cause inaccuracies in attenuation correction procedures and improper correlations of anatomy and function. All manufacturers recommend the scanning of an image registration phantom, such as the one shown in Figure 15, at a recommended weekly or monthly frequency. The phantom shown in Figure 15 has 6 radioactivity-filled syringes that are positioned along the 3 imaging axes. The phantom is scanned sequentially with the CT scanner and scintillation camera, and errors are calculated between the measured center locations of the syringes. These measurements are then compared with the acceptable errors for the system, typically $3-5 \mathrm{~mm}$, to verify that the registration accuracy is sufficient for clinical applications. It may be important to do these measurements with substantial weight on the table to mimic the clinical situation.

Currently, the most common application of attenuation correction techniques in nuclear medicine is cardiac perfusion imaging (13). In many patients, diaphragmatic attenuation of the inferior wall occurs often and makes it difficult to distinguish between ischemia and attenuation artifact in this region. The use of accurate attenuation correction can provide valuable information in making the correct diagnostic interpretation. However slight misalignments of the transmission and emission scans, often due to patient motion, can result in artifacts in the attenuation-corrected images. Because of this potential problem, manufacturers typically provide correction software to adjust the alignment of the 2 datasets. An example of currently available ACQC (Attenuation Correction Quality Control) software provided by

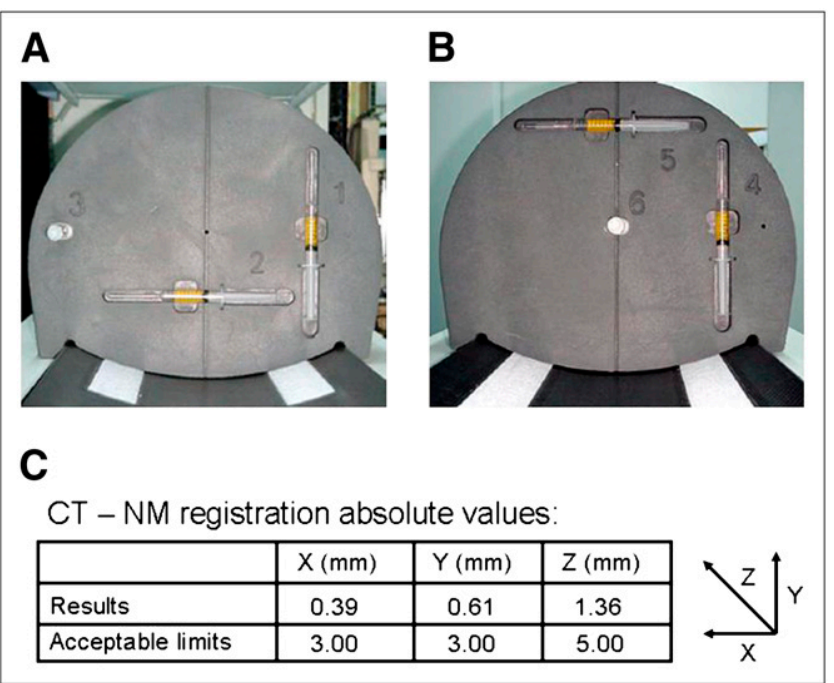

FIGURE 15. Front $(\mathrm{A})$ and back (B) views of SPECT/CT registration phantom and $(C)$ example of error evaluation performed with this phantom. 
A

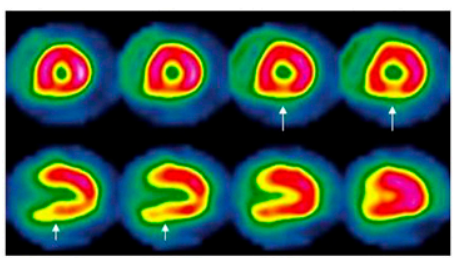

C

FIGURE 16. Demonstration of attenuation correction artifacts in perfusion study (A) resulting from misalignment of $C T$ and SPECT datasets $(B)$ and removal of these artifacts $(\mathrm{C})$ after realignment of datasets (D) using registration quality assurance software.

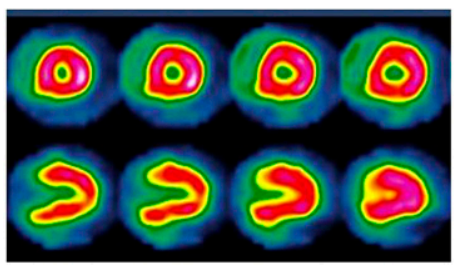

B

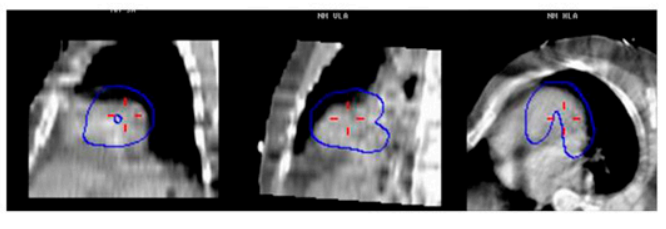

D

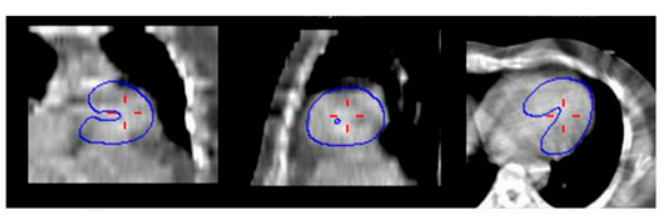

General Electric Healthcare Systems is shown in Figure 16. In Figure 16B, CT images of the myocardium in the standard format of 3 orthogonal views are shown with the perfusion images fused to this dataset and presented as contours typically set at $30 \%$ of maximum counts. In these images, there is an obvious misalignment that results in attenuationcorrected images with artifacts of decreased perfusion as shown in Figure 16A. Under cursor control, the datasets can be realigned in the 3 views resulting in correct alignment as shown in Figure 16D. This procedure produces accurate attenuation-corrected perfusion images (Fig. 16C). This is a valuable quality assurance technique that should be used regularly to validate registration before final image reconstruction and display to increase the level of confidence of the interpreting physician (14).

\section{CONCLUSION}

The combination of SPECT and CT systems has provided nuclear medicine physicians with a valuable tool in diagnosis and treatment planning by the integration of anatomic and functional images into a single registered dataset acquired in a single imaging session. These combined datasets provide increased levels of confidence not only in locating abnormal radiopharmaceutical distributions but also in differentiating between abnormal and normal uptake in body regions of complex anatomy. In addition, the combination of SPECT and CT provides the capability for accurate attenuation correction of measured radiopharmaceutical distributions. This application can eliminate attenuation artifacts from the diagnostic process when used in conjunction with routine quality assurance techniques to ensure proper registration of the 2 acquired datasets.

\section{REFERENCES}

1. Lang TF, Hasegawa BH, Liew SC, et al. Description of a prototype emissiontransmission computed-tomography imaging system. J Nucl Med. 1992;33: 1881-1887.

2. Cherry SR, Sorenson JA, Phelps ME. Tomographic reconstruction in nuclear medicine. In: Physics in Nuclear Medicine. 3rd ed. Philadelphia, PA: Saunders; 2003:273-297.

3. Cherry SR, Sorenson JA, Phelps ME. Single photon emission computed tomography. In: Physics in Nuclear Medicine. 3rd ed. Philadelphia, PA: Saunders; 2003:299-324.

4. Zaidi H, Hasegawa BH. Determination of the attenuation map in emission tomography. J Nucl Med. 2003;44:291-315.

5. X-ray computed tomography. In: Bushberg JT, Seibert JA, Leidholdt EM Jr, Boone JM eds. The Essential Physics of Medical Imaging. 2nd ed. Philadelphia, PA: Lippincott Williams and Wilkins; 2002:327-369.

6. Bruyant PP. Analytic and iterative reconstruction algorithms in SPECT. $\mathrm{J} \mathrm{Nucl}$ Med. 2002;43:1343-1358.

7. King MA, Glick SJ, Pretorius PH, et al. Attenuation, scatter, and spatial resolution compensation in SPECT. In: Wernick MN, Aarsvold JN, eds. Emission Tomography: The Fundamentals of PET and SPECT. London, U.K.: Elsevier Academic Press; 2004:473-494.

8. Kinahan PE, Hasegawa BH, Beyer T. X-ray-based attenuation correction for positron emission tomography/computed tomography scanners. Semin Nucl Med. 2003;23:166-179.

9. LaCroix KJ, Tsui BMW, Hasegawa BH, et al. Investigation of the use of x-ray CT images for attenuation compensation in SPECT. IEEE Trans Nucl Sci. 1994; NS-41:2793-2799.

10. Blankespoor SC, Xu X, Kalki CK, et al. Attenuation correction of SPECT using X-ray $\mathrm{CT}$ on an emission-transmission $\mathrm{CT}$ system: myocardial perfusion assessment. IEEE Trans Nucl Sci. 1996;NS-43:2263-2274.

11. Tharp K, Israel O, Hausmann J, et al. Impact of ${ }^{131} \mathrm{I}$-SPECT/CT images obtained with an integrated system in the follow-up of patients with thyroid carcinoma. Eur J Nucl Med Mol Imaging. 2004;31:1435-1442.

12. Turkington TG, Patton JA. Accuracy of SPECT attenuation correction using an x-ray attenuation map [abstract]. J Nucl Med. 2004;45(suppl): $416 \mathrm{P}$.

13. Computed tomographic imaging within nuclear cardiology, ASNC information statement-approved November 2004. J Nucl Cardiol. 2005;12:131142.

14. Tonge CM, Ellul G, Pandit M, et al. The value of registration correction in the attenuation correction of myocardial SPECT studies using low resolution computed tomography images. Nucl Med Commun. 2006;27:843-852. 